CLINICAL STUDY

\title{
Successful use of weekly pegvisomant administration in patients with acromegaly
}

\author{
C E Higham, J D J Thomas ${ }^{1}$, M Bidlingmaier ${ }^{2}$, W M Drake ${ }^{1}$ and P J Trainer \\ Department of Endocrinology, The Christie Hospital, Manchester M2O 4BX, UK, ${ }^{1}$ Department of Endocrinology, St Bartholomew's Hospital, London, UK \\ and ${ }^{2}$ Medizinische Klinik-Innenstadt, Ludwig-Maximilians Univeristat Munich, Munich, Germany \\ (Correspondence should be addressed to P J Trainer; Email: peter.trainer@manchester.ac.uk)
}

\begin{abstract}
Context: Clinical trials using $80 \mathrm{mg}$ once weekly pegvisomant (pegV) in active acromegaly led to a $30 \%$ fall in serum IGF1. Subsequent studies demonstrated that daily administration of up to $40 \mathrm{mg} / \mathrm{day}$ achieved an IGF1 within reference range in $97 \%$ of patients. PegV has a half-life of $>70 \mathrm{~h}$ suggesting weekly dosing may be possible but using higher doses than in the initial trials.

Objective: To determine the efficacy of weekly dosing of pegV.

Design: A two center, open-label prospective study in patients with acromegaly converted from a stable daily dose of pegV (median dose $15 \mathrm{mg}$ daily (range 10-20 mg od), IGF1 normal for 3 months prior to inclusion) to twice-weekly (week 0-16) followed by once-weekly (week 16-32) administration.

Results: Seven patients (4M, age $57 \pm 7$ years, $6 / 7$ prior transsphenoidal surgery, $7 / 7$ prior radiotherapy) were recruited. Six patients completed the twice-weekly and five patients both the twice-weekly and once-weekly administration. Headaches led to two patient withdrawals at $0+24$ weeks. Mean pre-dose serum IGF1 levels remained stable with the different administration regimens (IGF1 baseline $145 \pm 39 \mathrm{ng} / \mathrm{ml}$, twice-weekly $124 \pm 39 \mathrm{ng} / \mathrm{ml}$ and once-weekly $127 \pm 22 \mathrm{ng} / \mathrm{ml}$ ) and all values were within age adjusted IGF1 reference range. PegV dose was reduced in two patients and five opted to continue weekly administration at trial termination. Safety and quality of life parameters remained stable.

Conclusions: Twice and once-weekly administration of pegV is effective in controlling serum IGF1 levels in acromegaly and although not formally assessed, continuation of weekly dosing in five patients at study conclusion suggests patient preference for this regimen.
\end{abstract}

European Journal of Endocrinology 161 21-25

\section{Introduction}

Pegvisomant (pegV) is a $\mathrm{GH}$ receptor antagonist licensed as a daily subcutaneous injection for the treatment of acromegaly and is capable of normalizing insulin-like growth factor-1 (IGF1) in up to $97 \%$ of patients (1). However, as pegV has plasma half-life in excess of $70 \mathrm{~h}$ it is surprising that it is licensed as a once daily preparation (2). Indeed, initial phase I trials in healthy volunteers demonstrated that peak pegV levels occurred at $\sim 72 \mathrm{~h}$, with an IGF1 nadir at 5 days following a $1 \mathrm{mg} / \mathrm{kg}$ dose. The initial trials in patients with acromegaly (phase II) used weekly rather than daily administration. Eighty mg per week (the approximate equivalent of $1 \mathrm{mg} / \mathrm{kg}$ ) of pegV led to a $30 \%$ reduction in IGF1 but only $3 / 15$ patients achieved an IGF1 within the age-related reference range (3). This disappointing IGF1 normalization rate resulted in daily dosing being used in the definitive (phase III) study with up to $20 \mathrm{mg} /$ day achieving a serum IGF1 within reference range in $89 \%$ of patients $(1,4)$.
IGF1 normalization was achieved in $20 \%$ of patients using $80 \mathrm{mg}$ weekly in the phase II trials compared with $54 \%$ of patients administering the approximately equivalent $10 \mathrm{mg}$ daily (i.e. $70 \mathrm{mg}$ /week) in the phase III studies $(3,4)$. However, the inclusion criteria for these two studies differed (phase II; IGF $1>150 \%$ of upper limit of normal (ULN) versus phase III; IGF1 $>130 \%$ of ULN) and when the decrease in IGF1 is expressed as a percentage of baseline, it is equivalent between the weekly and daily dose regimens (27 and $31 \%$ respectively). The difference in normalization rates was therefore probably a result of the different baseline IGF1 values. These data suggest that weekly dosing may suffice but that the doses of pegV required to normalize IGF1 are greater than initially anticipated, leaving the question of whether daily administration is more efficacious unanswered.

There is evidence that less than daily administration of pegV is effective (5). Furthermore, the addition of weekly pegV at doses of up to $160 \mathrm{mg} /$ week in patients with acromegaly uncontrolled on maximum dose of 
somatostatin analogues alone was effective at normalizing IGF1 in all 32 patients $(6,7)$.

In this report, we describe a prospective study with 6 months open-label extension data investigating the efficacy of converting patients with a normal IGF1 from a stable dose of daily pegV (10-20 mg daily) to twice and once weekly administration.

\section{Methods and patients}

\section{Protocol}

A two center, open label, prospective study was carried out at the Christie Hospital, Manchester and St Bartholomew's Hospital, London with appropriate UK ethical and regulatory approval. Patients with acromegaly on a stable dose of sole pegV therapy with serum IGF1 within the reference range for 3 months were eligible for inclusion.

The daily pegV dose was converted to an equivalent total weekly amount. During the initial 16 weeks of the trial, pegV was self-administered twice-weekly at three and four day intervals. The second 16 weeks of the trial involved administration of the total dose on a weekly basis (i.e. a patient on $10 \mathrm{mg}$ daily prior to the trial administered 40 and $30 \mathrm{mg}$ twice-weekly and $70 \mathrm{mg}$ weekly). Patients were instructed to reconstitute the daily dose of pegV in the recommended volume of solute $(1 \mathrm{ml})$ and administer these as separate injections.

Serum IGF1 levels were monitored at weeks $0,6,16$, 24, and 32. During week 32, patients attended for daily serum IGF1, GH, and pegV levels (patients attended, where possible, in the morning at $24 \mathrm{~h}$ intervals following the weekly injection). All samples were analyzed at each visit and also as a single batch at trial termination to eliminate inter-assay variation.

At each visit, vital signs, fasting glucose, and liver function tests (ALT, alanine transaminase; AST, aspartate aminotransferase; ALP, alkaline phosphatase and gGT) were monitored and an AcroQol questionnaire completed (8).

\section{Patients' characteristics}

Seven patients (four males, age $57 \pm 7$ years) were recruited, six completed the twice-weekly protocol and five completed the whole 32 weeks. Baseline characteristics are shown in Table 1. All patients were converted to pegV following failure to normalize IGF1 on prior treatments and those with hypopituitarism were on stable replacement. Tumor size was monitored by an annual magnetic resonance imaging.

\section{Assays}

All samples for IGF1, GH, and pegV were analyzed as a single batch.

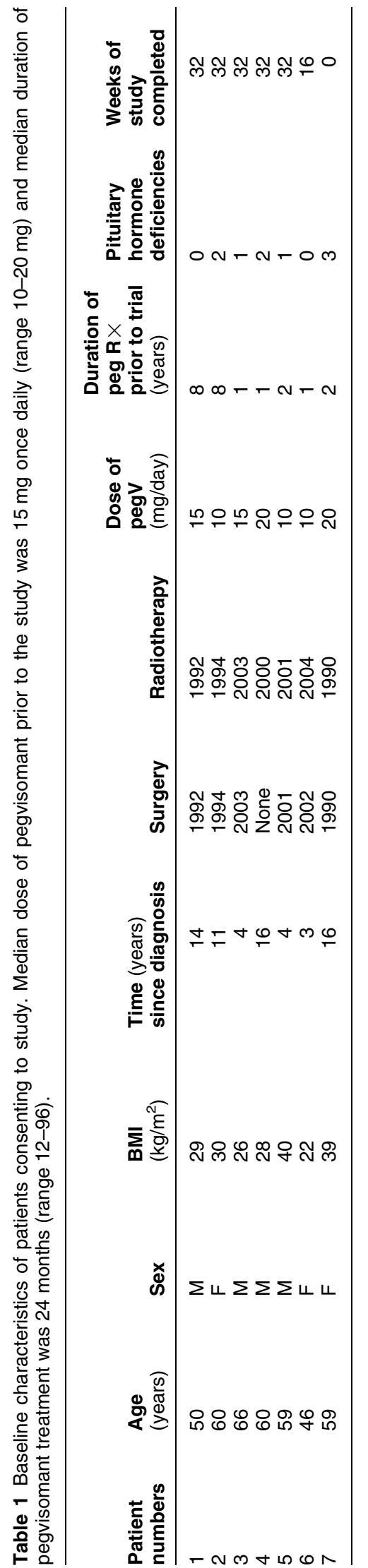


IGF1 Serum IGF1 levels were measured by Immulite2000 solid-phase enzyme-labeled chemiluminescent immunometric assay (DPC). Within assay variability was $6.4,2.9$, and $2.6 \%$ at low (mean $45.6 \pm 2.9 \mathrm{ng} / \mathrm{ml}$ ), medium (mean $238 \pm 6.9 \mathrm{ng} / \mathrm{ml}$ ), and high (mean $550 \pm 14.5 \mathrm{ng} / \mathrm{ml})$ serum IGF values respectively. The age-related IGF1 reference range was based on 1499 samples analyzed on the DPC Immulite assay from a healthy adult population (9).

PegV drug level Serum concentrations of pegV were determined by an immunofluorometric sandwich type assay previously described (10). Within assay variability was $7.5,4.6$, and $5.2 \%$ at concentrations of 160,650 , and $3900 \mathrm{ng} / \mathrm{ml}$ respectively. The between assay variability was $13.5,6.4$, and $8.5 \%$ at the same concentrations.

GH Serum concentrations of GH were determined using a specific $\mathrm{GH}$ assay designed to exclude interference from pegV. This assay has been described in detail elsewhere $(2,11)$. Intra-assay variability was 4.1 and $3.9 \%$ at concentrations of 5.2 and $14.6 \mathrm{ng} / \mathrm{ml}$ respectively. Inter-assay variability at the same concentrations was 7.3 and $9.2 \%$ respectively.

Liver function and plasma glucose Plasma glucose was measured using glucose oxidase method and ALT by Automated ADVIA system.

\section{Statistical analysis}

Data are normally distributed and expressed as mean \pm s.D. Serum IGF1 is expressed as an absolute concentration $(\mathrm{ng} / \mathrm{ml})$ and also as a percentage of the ULN for the age-related reference range.

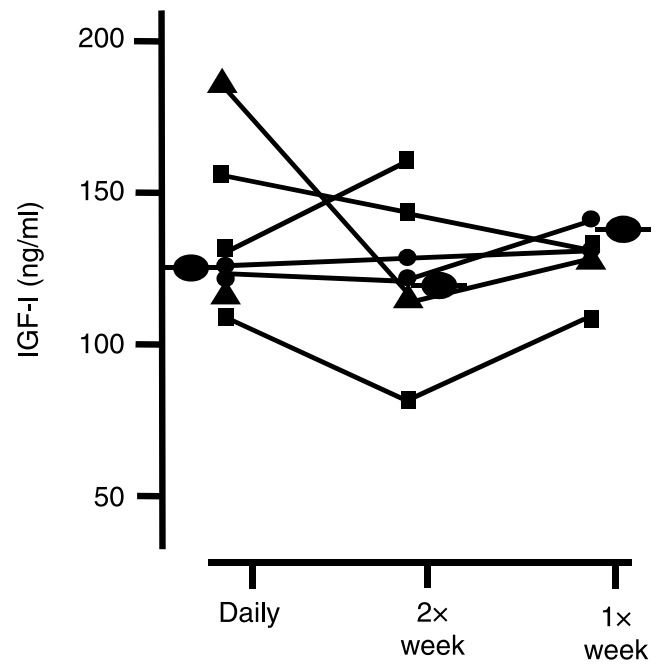

Figure 1 Trough serum IGF1 levels during each administration regime. $\Delta 20 \mathrm{mg} /$ day, $15 \mathrm{mg} /$ day, $10 \mathrm{mg} /$ day, $\bullet$ mean IGF1.
Longitudinal data were compared using RM-ANOVA. A paired $t$-test was used to analyze pre- and post-dose IGF1 and pegV values. Results were considered significant if $P<0.05$.

\section{Results}

\section{Mean serum IGF1 and pegV levels}

Mean serum IGF1 levels were unchanged during daily, twice-weekly or once-weekly administration of pegV $(139 \pm 27,124 \pm 26$, and $126 \pm 10 \mathrm{ng} / \mathrm{ml}$ respectively, RM-ANOVA, $P=0.6$ Fig. 1). Mean IGF1 values

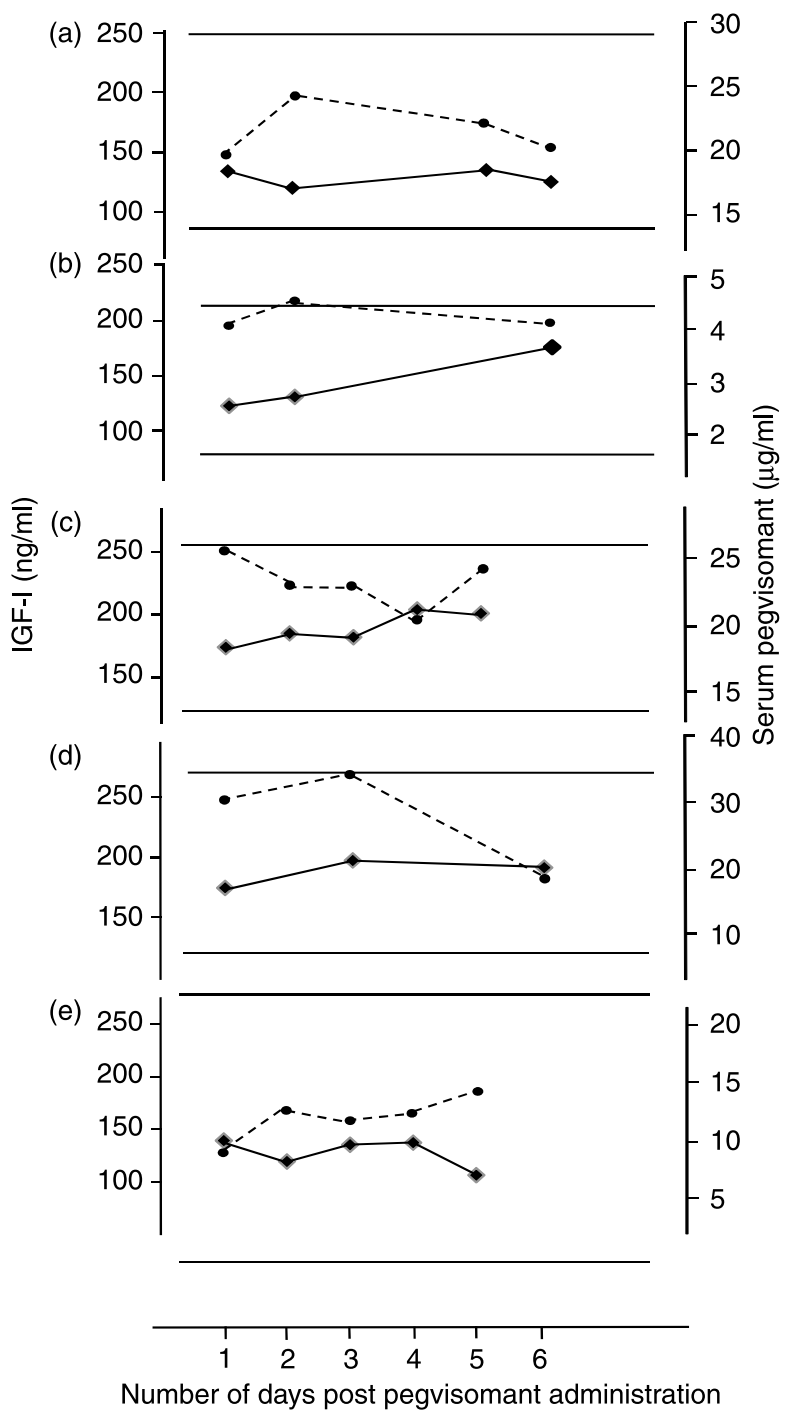

Figure 2 Serum IGF1 and pegvisomant levels in five patients $(\mathrm{a}-\mathrm{e})$ at week 32 . Samples were taken at $\sim 24 \mathrm{~h}$ intervals following the weekly injection: (b) and (e) $=70 \mathrm{mg} / \mathrm{week}$, (a) and (c) $=105 \mathrm{mg} /$ week and (d)=140 mg/week; (a) (c) and (d) = males, (b) and (e)=females. Upper and lower limits for the IGF1 reference ranges are indicated as horizontal lines. - Serum pegvisomant (dashed line); serum IGF1 (complete line). 
remained within reference range (IGF1 $61 \pm 14 \%$ of ULN at baseline, $54 \pm 10 \%$ ULN during twice-weekly and $56 \pm 10 \%$ ULN during once-weekly, Fig. 1). No dose adjustments in peg $\mathrm{V}$ were necessary.

There was no significant difference in mean serum pegV levels between daily, twice- and once-weekly

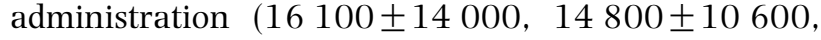
and $16900 \pm 9700 \mathrm{ng} / \mathrm{ml}$ respectively).

\section{Efficacy of weekly administration of pegV}

Serum IGF1 and pegV levels were measured one day post-dose and prior to the next dose in five patients during the weekly administration of pegV. There was no significant difference between serum IGF1 (post-dose $129 \pm 6.9$ versus pre-dose $137 \pm 22 \mathrm{ng} / \mathrm{ml}$ ) and pegV concentrations (post-dose $17200 \pm 11500$ versus predose $14900 \pm 6600 \mathrm{ng} / \mathrm{ml}$ ) although these decreased in four out of five patients over the week. Serum IGF1 values remained within reference range throughout the week following injection (Fig. 2). Serum GH levels showed no overall significant change (post-dose 22.6 \pm 24 versus pre-dose $29.5 \pm 36 \mathrm{ng} / \mathrm{ml}$; Fig. 3b) patients with the higher serum $\mathrm{GH}$ levels receiving the higher doses of pegV.

\section{Safety and metabolic data}

Serum ALT, fasting plasma glucose, and weight were unchanged throughout the study (ALT $29 \pm 11,25 \pm 6$, $29 \pm 11 \mathrm{IU} / \mathrm{l}$; fasting plasma glucose (FPG) $4.6 \pm 0.4$, $5.7 \pm 1.6,4.7 \pm 0.4 \mathrm{mmol} / \mathrm{l}$; weight $82 \pm 11,83 \pm 6,83$ $\pm 6 \mathrm{~kg}$ for daily, twice-weekly, and once-weekly respectively).

\section{Quality of life}

There was a trend to increase quality of life with conversion to less than daily dosing although this was not significant in this small number of patients (a)

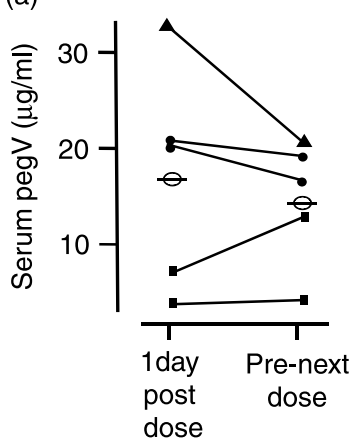

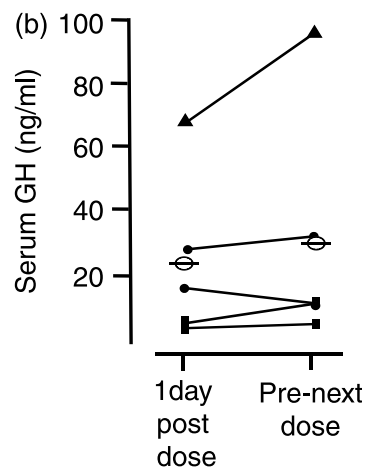

Figure 3 Serum pegvisomant (a) and GH concentrations (b) during weekly administration, 1 day post pegvisomant and prior to next dose. $\Delta 20 \mathrm{mg} /$ day, $15 \mathrm{mg} /$ day, $10 \mathrm{mg} /$ day, $\ominus$ mean IGF1.
(AcroQol scores $81 \pm 15,83 \pm 18,88 \pm 15$ for daily, twice-weekly, and once-weekly administration respectively $P>0.05)$.

\section{Adverse events}

Two patients did not complete the study (Table 1). Patient seven developed headaches following the first $60 \mathrm{mg}$ injection and declined to continue in the study. Patient six dropped out at the end of the twice-weekly administration due to headaches related to chronic sinusitis.

\section{Six month post-trial extension data}

All five patients completing the trial elected to continue with once-weekly administration of pegV. The dose of pegV was reduced to $60 \mathrm{mg}$ from $70 \mathrm{mg} /$ week in patient 5 and to $90 \mathrm{mg}$ from $105 \mathrm{mg} /$ week in patient 1 . Mean trough serum IGF1 levels were not significantly different 6 months post-trial on weekly dosing $(57 \pm 14$ vs 66 $\pm 11 \% \mathrm{ULN})$.

\section{Discussion}

This study illustrates for the first time that $\operatorname{peg} V$ as a sole therapy can be successfully administered as a weekly dose.

PegV was initially developed as a once-weekly preparation based on a half-life of over $70 \mathrm{~h}$. PegV is a relatively inefficient competitive receptor antagonist as plasma concentrations $\sim 1000$-fold higher than the GH are required to antagonize its action, which led to an initial underestimation of the dose that would be required to achieve normalization of IGF1. The importance of ensuring IGF1 normalization led to the use of a daily dosing strategy in the landmark pegV clinical trial and licensing as a daily preparation.

Studies using daily administration and individualized dose titration strategies demonstrate the wide range of doses, from 10 to $60 \mathrm{mg} /$ day that may be required to normalize IGF1. We have demonstrated that it is possible to convert patients from daily dose requirements of up to $20 \mathrm{mg} /$ day (between 70 and $140 \mathrm{mg}$ of pegV per week) to a weekly administration regimen with no loss of efficacy with regards to serum IGF1 levels. This was maintained at 6 months after the end of the study, where all patients elected to continue the weekly dose. The number of patients in this study was small and therefore small differences in IGF1 levels between the different regimens cannot be excluded. From a practical point of view, however, all the individual IGF1 levels remained below the ULN with no requirement for an increase in pegV dose during the study or its extension.

All patients in this study had previously been treated with radiotherapy. It is known that radiotherapy minimally reduces the dose of pegV required to 
normalize IGF1 (12) and that radiotherapy continues to reduce $\mathrm{GH}$ levels for up to 15 years post-treatment. Therefore we cannot completely exclude that the ongoing effect of radiotherapy may have contributed to the sustained control of IGF1 on conversion to weekly dosing. However, there was no evidence of a fall in circulating GH levels as would be anticipated if radiotherapy was a factor in the successful conversion to a weekly dose of pegV.

As would be expected in the case of a competitive receptor antagonist, maintaining serum levels are crucial to efficacy; in this study, serum pegV levels dropped in four out of five patients over the weekly administration period but these levels were still adequate to maintain an IGF1 within the age-related reference range. This study was not a pharmacokinetic study but three of the patients had a peak serum pegV level at 2-3 days post-pegV, and the single patient with an increased pegV level towards the end of the week remains unexplained. There was no overall significant change in pegV levels, which may indicate that the half-life of pegV is greater than the $72 \mathrm{~h}$ originally calculated, but detailed pharmacokinetic studies are required to address this. Serum GH levels remained stable on pegV over the weekly administration and were higher in those with greater serum pegV levels, reflecting the dose of pegV required to normalize IGF1.

All patients elected to continue the weekly administration frequency at the end of the study despite the same overall total number of injections and large volume of injection (up to $7 \mathrm{ml}$ ) required. The lack of a weekly formulation for pegV is the biggest obstacle to this administration regimen. We would predict that a weekly formulation involving the reduced number and volume of injections would improve patient satisfaction still further.

To conclude, we have demonstrated that conversion to sole pegV administration on a weekly basis can be achieved safely, with no loss of efficacy or increase in dose requirements and, although not formally assessed, continuation of weekly dosing in five patients at study conclusion suggests patient preference for this regimen.

\section{Declaration of interest}

P J Trainer and W M Drake have received research funding and honoraria from Pfizer.

\section{Funding}

This study was supported by an independent investigator grant from Pfizer.

\section{Acknowledgements}

We would like to acknowledge the expert technical help of Margaret Roberts and Kathy Maher.

\section{References}

1 van der Lely AJ, Hutson RK, Trainer PJ, Besser GM, Barkan AL, Katznelson L, Klibanski A, Herman-Bonert V, Melmed S, Vance ML, Freda PU, Stewart PM, Friend KE, Clemmons DR, Johannsson G, Stavrou S, Cook DM, Phillips LS, Strasburger CJ, Hackett S, Zib KA, Davis RJ, Scarlett JA \& Thorner MO. Long-term treatment of acromegaly with pegvisomant, a growth hormone receptor antagonist. Lancet 2001358 1754-1759.

2 Thorner MO, Strasburger CJ, Wu Z, Straume M, Bidlingmaier M, Pezzoli SS, Zib K, Scarlett JC \& Bennett WF. Growth hormone (GH) receptor blockade with a PEG-modified GH (B2036-PEG) lowers serum insulin-like growth factor-I but does not acutely stimulate serum GH. Journal of Clinical Endocrinology and Metabolism 1999 84 2098-2103.

3 Van der Lely AJ, Lamberts SWJ, Barkan A, Pandya N, Besser GM, Trainer PJ, Bonert V, Melmed S, Clemmons DR, Rose R, Vance ML, Thorner MO, Zib KA, Davis RJ, Bennett WF, Scarlett JA. A six week, double blind, placebo controlled study of a growth hormone antagonist B2036-PEG (Trovert $^{\mathrm{TM}}$ ), in acromegalic patients. Program of the 80th annual meeting of the Endocrine Society, New Orleans, LA p57 (Abstract OR4-1) 1998.

4 Trainer PJ, Drake WM, Katznelson L, Freda PU, Herman-Bonert V, van der Lely AJ, Dimaraki EV, Stewart PM, Friend KE, Vance ML, Besser GM, Scarlett JA, Thorner MO, Parkinson C, Klibanski A, Powell JS, Barkan AL, Sheppard MC, Malsonado M, Rose DR, Clemmons DR, Johannsson G, Bengtsson BA, Stavrou S, Kleinberg DL, Cook DM, Phillips LS, Bidlingmaier M, Strasburger CJ, Hackett S, Zib K, Bennett WF \& Davis RJ. Treatment of acromegaly with the growth hormone-receptor antagonist pegvisomant. New England Journal of Medicine 200020 1171-1177.

5 Jehle S, Reyes CM, Sundeen RE \& Freda PU. Alternate day administration of pegvisomant maintains normal serum insulinlike growth factor-I levels in patients with acromegaly. Journal of Clinical Endocrinology and Metabolism 200590 1588-1593.

6 Feenstra J, de Herder WW, ten Have SM, van den Beld AV, Feelders RA, Janssen JA \& van der Lely AJ. Combined therapy with somatostatin analogs and weekly pegvisomant in active acromegaly. Lancet 2005365 1644-1646.

7 Neggers S, van Aken MO, Janssen J, Feelders RA, de Herder WW \& ven der Lely AJ. Long-term efficacy and safety of combined treatment of somatostatin analogs and pegvisomant in acromegaly. Journal of Clinical Endocrinology and Metabolism 200792 4598-4601.

8 Rowles SV, Prieto L, Badia X, Shalet SM, Webb SM \& Trainer PJ. Quality of life (QOL) in patients with acromegaly is severely impaired: use of a novel measure of QOL: acromegaly quality of life questionnaire. Journal of Clinical Endocrinology and Metabolism $2005903337-3341$.

9 Elmlinger MW, Kühnel W, Weber MM \& Ranke MB. Reference ranges for two automated chemiluminescent assays for serum insulin-like growth factor I (IGF1) and IGF-binding protein 3 (IGFBP-3). Clinical Chemistry and Laboratory Medicine $2004 \mathbf{4 2}$ 654-664.

10 Roemmler J, Bidlingmaier M \& Schopol J. Endogenous estradiol may influence IGF1 levels in acromegalic women treated with pegvisomant. Pituitary, 2008. In Press, DOI: 10.1007/s11102-008-0131-5.

11 Muller AF, Janssen JA, Hofland LJ, Lamberts SW, Bidlingmaier M, Strasburger CJ \& van der Lely AJ. Blockade of the growth hormone (GH) receptor unmasks rapid GH-releasing peptide-6-mediated tissue-specific insulin resistance. Journal of Clinical Endocrinology and Metabolism 200186 590-593.

12 Parkinson C, Burman P, Messig M \& Trainer PJ. Gender, body weight, disease activity and previous radiotherapy influence the response to pegvisomant. Journal of Clinical Endocrinology and Metabolism 200792 190-195.

Received 3 March 2009

Accepted 1 April 2009 